\title{
POLARIMETRY IN THE OUTSKIRTS OF NGC $6611^{1}$
}

\author{
Ana M. Orsatti, ${ }^{2,3}$ E. Irene Vega, ${ }^{3,4}$ and Hugo G. Marraco ${ }^{3}$ \\ Facultad de Ciencias Astronómicas y Geofísicas, Universidad Nacional de La Plata, Paseo del Bosque s/n, \\ B1900FWA La Plata, Argentina; hmarraco@fcaglp.unlp.edu.ar \\ Received 2005 October 21; accepted 2006 July 8
}

\begin{abstract}
We present new polarimetric UBVRI observations of 25 stars in the direction of the halo of NGC 6611, the rich stellar open cluster embedded in an ionized hydrogen complex (M16). Our plan is to characterize the interstellar material (ISM) associated with halo stars in order to make a comparison with the ISM dusty core characteristics that resulted from a previous investigation by the same authors. Of the halo stars, $47 \%$ ( 8 out of 17 ) show indications of intrinsic polarization in their light, similar to what was found for core stars $(50 \%)$. We have identified the presence of nearby dust clouds located on the Local arm that produce a mean polarization of about $1 \%$; a value $\overline{\lambda_{\max }}=$ $0.61 \pm 0.07 \mu \mathrm{m}$, which is slightly larger than that of the average ISM; and a mean direction of the polarization vectors of $\overline{\theta_{V}}=81.9 \pm 1.8$. The ISM associated with the halo region has $\overline{\lambda_{\max }}$ similar to the general interstellar medium $(0.55 \pm 0.07 \mu \mathrm{m})$. The observed polarizations show a gradual increase from halo $\left(\overline{P_{\max }}=1.93 \% \pm 0.3 \%\right)$ to core $\left(\overline{P_{\max }}=3.19 \% \pm 0.63 \%\right)$. Position angles of the $e$-vector for both groups are generally similar, but there exists a slight difference in mean direction between them that is within the errors. We have also found that the halo stars are possibly represented by Whittet \& van Breda's relationship, while in the cluster's core the dust does not fulfill the abovementioned relationship. As a conclusion, we cannot find any clear difference between core and halo dust characteristics, with the exception of $\overline{\lambda_{\max }}$, which may suggest a change in dust size.
\end{abstract}

Key words: dust, extinction - open clusters and associations: individual (NGC 6611) - techniques: polarimetric

Online material: machine-readable table

\section{INTRODUCTION}

NGC 6611 (C1816-138) is a stellar rich open cluster located in the Sagittarius spiral arm in the plane of the Galaxy $\left(l=17^{\circ} .0, b=0^{\circ} 8\right)$. It is embedded in an ionized hydrogen complex (M16), a large nebulosity connected with dense dust clouds. The central portion of the cluster is part of the northwestern part of M16, but nowadays it is assumed that it comprises the whole complex (e.g., de Winter et al. 1997).

This object and the surrounding dark and bright nebulosities have been the subject of a great number of studies, centered particularly on two main issues: the existence of an age spread (proposed by Hillenbrand et al. [1993] and confirmed by de Winter et al. [1997]) and also the determination of which extinction law is valid for the cluster.

Several years ago, Orsatti et al. (2000, hereafter OVM00) conducted an investigation to independently test the existence of larger than average grains in the dusty central part of NGC 6611. Through UBVRI polarimetric observations, they found that the Whittet \& van Breda (1978) relation between $E_{V-K} / E_{B-V}$ and $\lambda_{\max }$ (the wavelength of maximum interstellar polarization) is not valid for stars belonging to the M16 core. This situation may arise mainly from the presence of silicate grains of slightly larger size than the standard interstellar material (ISM) and also from a considerable increase in mean graphite grain size, according to

\footnotetext{
${ }^{1}$ Based on observations obtained at Complejo Astronómico El Leoncito, operated under agreement between the Consejo Nacional de Investigaciones Científicas y Técnicas de la República Argentina and the Universities of La Plata, Córdoba, and San Juan.

${ }^{2}$ Instituto de Astrof ísica de La Plata, CONICET, Paseo del Bosque s/n, 1900 La Plata, Argentina.

${ }^{3}$ Member of the Carrera del Investigador Científico, CONICET.

${ }^{4}$ Instituto de Astronomía y Física del Espacio, CONICET, C.C. 67, Sucursal 28, 1428 Buenos Aires, Argentina.
}

previous results from Chini \& Wargau (1990). It was also found by OVM00 that a high percentage of the observed stars (about $50 \%$ ) displayed indications of intrinsic polarization in their measurements, a percentage similar to that found in IC 2944 (Vega et al. 1994), another young open cluster closely related to an $\mathrm{H}$ II region.

Since only stars in the core of NGC 6611 were observed in the OVM00 investigation, we propose here to extend the polarimetric observations to members belonging to the outskirts of the object. Our plan is to characterize the ISM associated with halo stars in order to make a comparison with the ISM dusty core characteristics. We also plan to isolate those stars with indications of a noninterstellar polarization in order to make a comparison between both sections of the cluster. To obtain a homogeneous set of observations, we have used the same photopolarimeter and technical reduction as in the past investigation.

\section{OBSERVATIONS}

Observations in the $U B V(R I)_{\mathrm{KC}}$ bands (Kron-Cousins; $\lambda_{U_{\mathrm{eff}}}=$ $0.36 \mu \mathrm{m}, \mathrm{FWHM}=0.05 \mu \mathrm{m} ; \lambda_{B_{\mathrm{cff}}}=0.44 \mu \mathrm{m}, \mathrm{FWHM}=0.06 \mu \mathrm{m}$; $\lambda_{V_{\text {eff }}}=0.53 \mu \mathrm{m}, \mathrm{FWHM}=0.06 \mu \mathrm{m} ; \lambda_{R_{\mathrm{eff}}}=0.69 \mu \mathrm{m}, \mathrm{FWHM}=$ $0.18 \mu \mathrm{m} ; \lambda_{I_{\text {eff }}}=0.83 \mu \mathrm{m}, \mathrm{FWHM}=0.15 \mu \mathrm{m}$ ) were carried out using the five-channel photopolarimeter of the Torino Astronomical Observatory attached to the $2.15 \mathrm{~m}$ telescope at the Complejo Astronómico El Leoncito (San Juan, Argentina). They were performed on three nights (June 14-16) in 2002 and one night (May 29) in 2003. Standard stars for null polarization and for the zero point of the polarization position angle were observed several times each night for calibration purposes, and the standards were taken from Clocchiati \& Marraco (1988). For further information on the instrument, data acquisition, and data reduction, see Scaltriti (1994).

Table 1 lists the 25 stars observed in the direction of the halo of the open cluster, including the percentage polarization $\left(P_{\lambda}\right)$, the 
TABLE 1

Polarimetric Observations in NGC 6611

\begin{tabular}{|c|c|c|c|c|c|c|c|}
\hline Star ${ }^{a}$ & $\mathrm{HD} / \mathrm{BD} / \mathrm{H}^{\mathrm{b}}$ & Filter & $\begin{array}{c}P_{\lambda} \\
(\%)\end{array}$ & $\begin{array}{c}\epsilon_{p} \\
(\%)\end{array}$ & $\begin{array}{c}\theta_{\lambda} \\
(\mathrm{deg})\end{array}$ & $\begin{array}{c}\epsilon_{\theta} \\
(\mathrm{deg})\end{array}$ & $n^{\mathrm{c}}$ \\
\hline \multirow[t]{5}{*}{$2 \ldots \ldots \ldots \ldots$} & \multirow[t]{10}{*}{ BD -134914 } & $U$ & 1.70 & 0.25 & 63.9 & 2.1 & 2 \\
\hline & & $B$ & 2.18 & 0.21 & 65.0 & 1.4 & \\
\hline & & $V$ & 1.99 & 0.13 & 72.5 & 0.9 & \\
\hline & & $R$ & 1.55 & 0.12 & 72.6 & 1.1 & \\
\hline & & $I$ & 1.59 & 0.19 & 71.1 & 1.7 & \\
\hline \multirow[t]{5}{*}{$25^{*} \ldots \ldots \ldots \ldots$} & & $U$ & 4.33 & 0.39 & 65.4 & 2.6 & 3 \\
\hline & & $B$ & 5.31 & 0.28 & 66.5 & 1.5 & \\
\hline & & $V$ & 5.49 & 0.07 & 61.4 & 0.3 & \\
\hline & & $R$ & 5.56 & 0.15 & 62.6 & 0.8 & \\
\hline & & $I$ & 5.58 & 0.17 & 63.4 & 0.4 & \\
\hline
\end{tabular}

NotE.-Table 1 is published in its entirety in the electronic edition of the Astronomical Journal. A portion is shown here for guidance regarding its form and content.

a Identifications from Walker (1961). An asterisk indicates a nonmember.

b Identifications from (H) Hoag et al. (1961), (HD) Henry Draper Catalog, or (BD) Bonner Durchmusterung.

c Number of integrations.

position angle of the electric vector $\left(\theta_{\lambda}\right)$ in the equatorial coordinate system, and their respective mean errors for each filter. We also indicate the number of $60 \mathrm{~s}$ independent integrations with each filter. Star identifications are taken from Walker (1961), and we indicate possible nonmembers, according to de Winter et al. (1997), with an asterisk in Table 1, as well as in Table 2. The distribution of the observed stars in the field of NGC 6611 is shown in Figure 1, where the rectangular area indicates the region studied by OVM00.

\section{RESULTS}

By observing the amount of interstellar polarization in several bandpasses, the wavelength at which maximum polarization $\left(P_{\max }\right)$ occurs can be computed. This wavelength, $\lambda_{\max }$, is a function of the optical properties and characteristic particle size distribution of the aligned grains (McMillan 1978; Wilking et al. 1980). The maximum polarization $P_{\max }$ has been calculated by fitting the observed polarization in the $U B V R I$ bandpasses to the standard Serkowski polarization law (Serkowski 1973),

$$
P_{\lambda} / P_{\max }=\exp \left[-K \ln ^{2}\left(\lambda_{\max } / \lambda\right)\right]
$$

and adopting the relationship

$$
K=-0.01+1.66 \lambda_{\max },
$$

with $\lambda_{\max }$ in microns, from Whittet et al. (1992).

If the polarization is well represented by this relation (meaning that the measured polarization is of interstellar origin), $\sigma_{1}$ (the unit weight error of the fit) should not be higher than 1.5 because of the weighting scheme; a higher value could indicate the presence of intrinsic polarization in the light from the star, i.e., a polarization that did not originate in the ISM between the star and the Sun. The dominant source of intrinsic polarization is asymmetric circumstellar dust (e.g., in binary systems) and, for classical Be stars, electron scattering.

Table 2 lists the $P_{\max }, \lambda_{\max }$, and $\sigma_{1}$ values for the observed stars. In the last column we also list spectral types from the WEBDA database $^{5}$ (developed and maintained by J. C. Mermilliod). The

\footnotetext{
${ }^{5}$ Available at http://obswww.unige.ch/webda.
}

TABLE 2

\begin{tabular}{|c|c|c|c|c|c|c|}
\hline $\operatorname{Star}^{\mathrm{a}}$ & $\begin{array}{c}P_{\max } \\
(\%)\end{array}$ & $\begin{array}{c}\epsilon_{p} \\
(\%)\end{array}$ & $\sigma_{1}^{\mathbf{b}}$ & $\begin{array}{c}\lambda_{\max } \\
(\mu \mathrm{m})\end{array}$ & $\begin{array}{c}\epsilon_{\lambda} \\
(\mu \mathrm{m})\end{array}$ & Spectral Type $^{c}$ \\
\hline $2 .$. & 1.96 & 0.14 & 1.14 & 0.44 & 0.06 & $\mathrm{~B} 8 \mathrm{~V}$ \\
\hline $25^{*}$. & 5.75 & 0.10 & 1.52 & 0.64 & 0.03 & $\mathrm{~B} 0.5 \mathrm{~V}$ \\
\hline $90 \ldots \ldots$ & 2.29 & 0.07 & 0.79 & 0.64 & 0.04 & B3 V \\
\hline $103^{*} .$. & 1.78 & 0.15 & 1.27 & 0.63 & 0.12 & $\mathrm{~A} 0 \mathrm{~V}$ \\
\hline $117 \ldots \ldots \ldots \ldots \ldots \ldots$ & 1.85 & 0.10 & 1.34 & 0.54 & 0.05 & $\ldots$ \\
\hline $161^{*} .$. & 3.21 & 0.58 & 0.49 & 0.28 & 0.01 & $08.5 \mathrm{~V}$ \\
\hline $184^{*}$. & 1.70 & 0.16 & 0.73 & 0.36 & 0.04 & K2 III \\
\hline $206^{*}$. & 0.84 & 0.02 & 0.31 & 0.67 & 0.02 & G6 II \\
\hline $222^{*}$ & 5.91 & 0.21 & 0.89 & 0.72 & 0.04 & O7 III ((f)) \\
\hline $255 \ldots$ & 2.62 & 0.28 & 1.16 & 0.31 & 0.04 & $\ldots$ \\
\hline $265 \ldots$ & 1.81 & 0.06 & 0.53 & 0.43 & 0.02 & $\ldots$ \\
\hline $290 \ldots$. & 1.56 & 0.04 & 1.64 & 0.53 & 0.04 & $\mathrm{~B} 1 \mathrm{~V}$ \\
\hline $340 \ldots$ & 2.09 & 0.14 & 1.99 & 0.50 & 0.07 & $\mathrm{~A} 0 \mathrm{~V}$ \\
\hline $344^{*}$. & 1.15 & 0.06 & 0.51 & 0.63 & 0.07 & $\ldots$ \\
\hline $407 \ldots$ & 1.71 & 0.05 & 1.20 & 0.61 & 0.04 & $\ldots$ \\
\hline $409 \ldots$ & 1.61 & 0.26 & 3.46 & 0.54 & 0.20 & $\mathrm{~B} 4 \mathrm{Ve}$ \\
\hline $417 \ldots$ & 1.51 & 0.04 & 0.96 & 0.53 & 0.02 & $\ldots$ \\
\hline $432^{*}$. & 0.96 & 0.03 & 0.58 & 0.50 & 0.02 & $\ldots$ \\
\hline $440^{*}$ & 1.49 & 0.23 & 2.46 & 0.49 & 0.04 & G1 III/K1 III \\
\hline $468 \ldots$ & 1.25 & 0.06 & 1.60 & 0.49 & 0.03 & $\mathrm{~B} 1 \mathrm{Vp}$ \\
\hline $469 \ldots$ & 1.81 & 0.03 & 1.56 & 0.63 & 0.02 & B1.5 Vpe \\
\hline $489 \ldots$ & 2.09 & 0.09 & 1.55 & 0.56 & 0.05 & B8.5 V \\
\hline . & 1.86 & 0.02 & 0.40 & 0.57 & 0.02 & B2 V (e?) \\
\hline $501 \ldots \ldots \ldots \ldots \ldots \ldots \ldots$ & 1.45 & 0.08 & 1.70 & 0.61 & 0.10 & $\ldots$ \\
\hline $520 \ldots \ldots$ & 2.36 & 0.05 & 2.50 & 0.27 & 0.09 & B7: V \\
\hline
\end{tabular}

Polarization Results

a Identifications from Walker (1961). An asterisk indicates a nonmember.

${ }^{b}$ Here $\sigma_{1}^{2}=\sum\left(r_{\lambda} / \epsilon_{p_{1}}\right)^{2} /(m-2)$, where $m$ is the number of colors and $r_{\lambda}=$ $P_{\lambda}-P_{\max } \exp \left[-K \ln ^{2}\left(\lambda_{\max } / \lambda\right)\right]$.

c Spectral type from the WEBDA database.

mathematical expression used to obtain the individual $\sigma_{1}$ values is found in this table as a footnote.

\section{ANALYSIS AND DISCUSSION}

From their measurements, 10 objects have values for the unit weight error of the fit $\left(\sigma_{1}\right)$ above 1.5. They are two nonmembers (stars 25 and 440) and eight members (stars 290, 340, 409, 468, $469,489,501$, and 520). As stated previously, these stars may be suspected of having intrinsic polarization. There is another criterion to detect this: a fitted $\lambda_{\text {max }}$ that is much shorter than the average general value for the interstellar medium $(0.55 \mu \mathrm{m}$; Serkowski et al. 1975); this is the case for star 161, another nonmember star, with a $\lambda_{\max }$ value that amounts to $0.28 \mu \mathrm{m}$.

The most remarkable $P_{\lambda}$ and $\theta_{\lambda}$ versus $\lambda$ plots for stars with indications of intrinsic polarization are shown in Figure 2, where the solid curve denotes the standard Serkowski curve (eq. [1]), the valid law for an interstellar origin for the measured polarization. The presence of intrinsic polarization in the light from a star causes a mismatch between observations and the Serkowski curve and/or a rotation in the position angle of the polarization vector. The above-mentioned mismatch is clearly seen in every plot, except for star 161, but the rotation in position angle with $\lambda$ is a common characteristic of the whole group of stars with $\sigma_{1}$ higher than 1.5 , including that star.

We have searched the literature looking for clues about possible origins of the intrinsic polarization detected in this group of 11 stars. We have found, for example, that Hillenbrand et al. (1993) suggest that star 25 (B0.5 V) has circumstellar material based on IR excesses, while Dûchene et al. (2001) have found that it is a member of a binary system, with a separation of 1.43 . The light from this star shows a very high polarization (5.75\%). According 


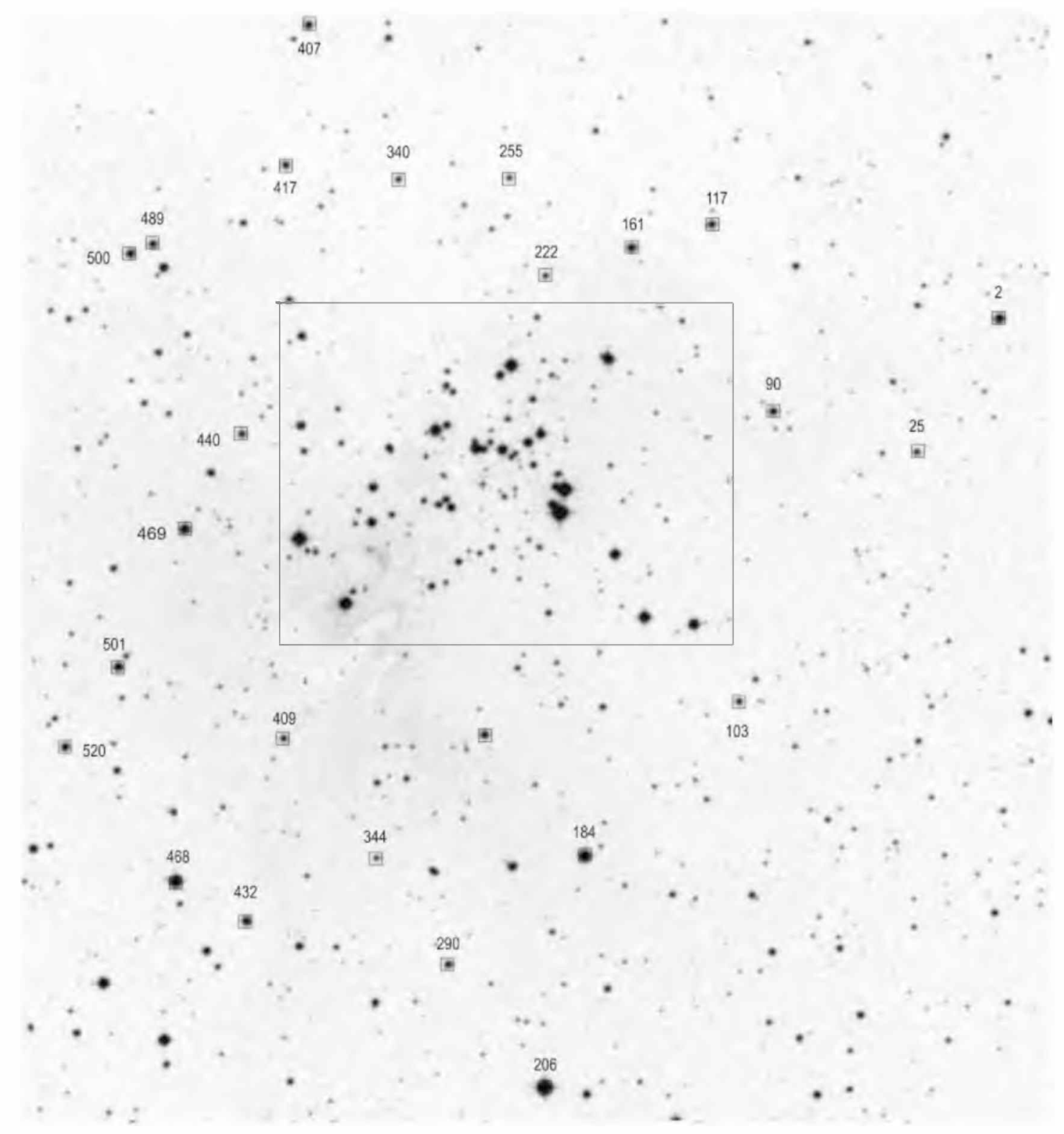

FIG. 1.-Stars observed in the field of NGC 6611. The rectangular area indicates the region studied by OVM00.

to the same authors, $\operatorname{star} 290\left(\mathrm{~B} 1 \mathrm{~V} ; \sigma_{1}=1.64\right)$ is part of another binary system, with a separation of 1 1"37 between members, but this fact cannot be associated with intrinsic polarization. Star 409 is an Orion-type variable with spectral type B4 Ve and a very high $\sigma_{1}$ value, 3.46. For stars 468 (B1 Vp) and 161, Kumar et al. (2004) have found that they show mid-IR $(7-100 \mu \mathrm{m})$ excesses, which indicate that cool circumstellar dust is present. This identifies them as Vega-like stars or precursors to such phenomena. Star 469 (B1.5 $\mathrm{Vp}$ ) has $\mathrm{H} \beta$ partially filled in with emission. We could not find any particular clue for the rest; they are probably undetected close binary systems or perhaps rapid rotators. The spectroscopic information used in this section comes from the WEBDA database.

It is known that for the interstellar medium the polarization efficiency (ratio of the maximum amount of polarization to visual extinction) rarely exceeds the empirical upper limit,

$$
P_{\max } \leq 3 A_{V} \simeq 3\left(R_{V} E_{B-V}\right) .
$$

obtained for interstellar dust particles (Hiltner 1956). The ratio $P_{\max } /\left(R_{V} E_{B-V}\right)$ depends mainly on the alignment efficiency and the magnetic field strength, as well as on the fact that sometimes radiation traverses more than one cloud with different field directions.

Figure 3 depicts the relation that exists between reddening and polarization originated in dust along the line of sight to NGC 6611. Open circles represent the halo member stars observed in this work, while filled circles are used for stars in the core (OVM00). Triangles represent the positions of the "front-side" stars, used to subtract the effects of foreground extinction from the light coming from any other star observed in the central region.

The empirical upper limit (eq. [2]), known as the line of maximum efficiency, has been drawn adopting $R_{V}=3.1$. Excesses $E_{B-V}$ were obtained from the literature or from the relationship between spectral type and color indices following Schmidt-Kaler (1982). It can be seen that the points lie to the right of the interstellar maximum line; this situation indicates that the observed polarization is mostly due to ISM.

Star 184 is located very close to the interstellar maximum line. It has been classified as K2 III ( Walker 1961), and its excess is low: $E_{B-V}=0.20 \mathrm{mag}$. Tucholke et al. (1986) have proposed a $0 \%$ membership probability in NGC 6611 for this star. Its 

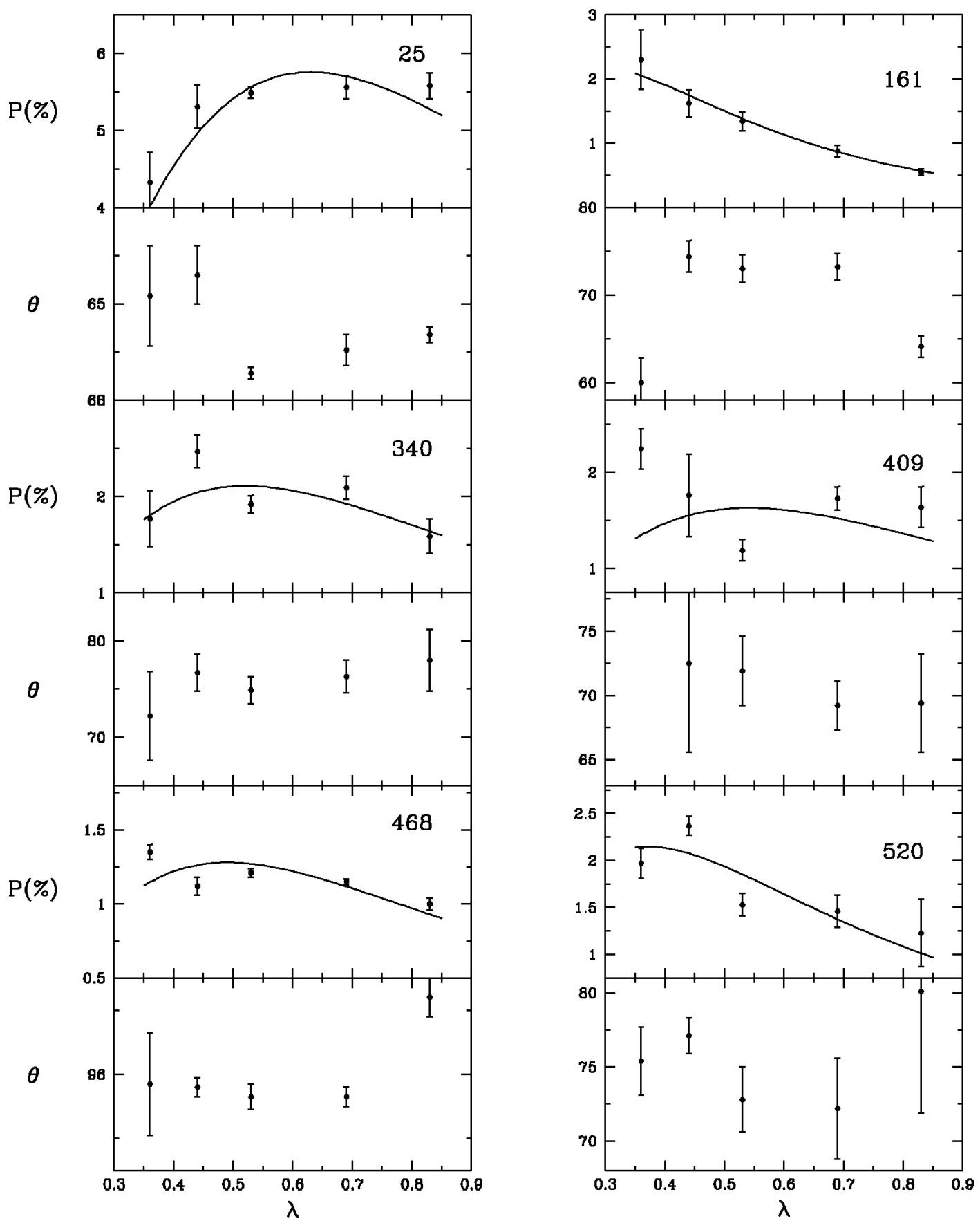

FIG. 2.- Polarization and position angle dependence on wavelength for stars with indications of intrinsic polarization (mismatch between observations and Serkowski curve and/or variable position angle).

polarization amounts to $P_{\max }=1.70 \%$, which is too high for its distance from the Sun $(534 \mathrm{pc})$. Even when the $\sigma_{1}$ value $(0.73)$ does not indicate the presence of intrinsic polarization, we think that due to evolution the star is ejecting material into space, from which the high polarization originates. Stars 25 and 222 are background stars according to the literature.

Figure 4 shows the run of the polarization vector $\boldsymbol{P}$ through its components in the equatorial system, the Stokes parameters $Q$ and $U$. This plot supplies useful information on variations in interstellar environments: if the light from individual members of an open cluster has gone through a common sheet of dust, their representative points will concentrate on a given region of the plot, indicating similar optical characteristics of the ISM. If the plot shows more than one concentration, this means that the light from a certain group of stars has traveled through different regions of dust with somewhat different characteristics. In this figure we identify at least two different groupings. One of them consists of the front-side stars 206, 344, and 432 (Fig. 4, squares), which belong to the Local arm. Based on the spectral type of star 206 we conclude that the light from this group is affected by nearby clouds, located less than $750 \mathrm{pc}$ from the Sun, which originate a mean polarization of about $1 \%$. Its $\overline{\lambda_{\max }}=0.61 \pm 0.07 \mu \mathrm{m}$ (mean of three stars) is slightly larger than that of the average ISM, and it has a mean direction of the polarization vectors of $\overline{\theta_{V}}=81^{\circ} .9 \pm$ $1^{\circ} .8$. The square plotted in the upper part of Figure 4 represents star 222 , previously mentioned as a background star.

The second group consists of halo member stars ( Fig. 4, open symbols) and core members ( filled symbols). Halo and core stars 


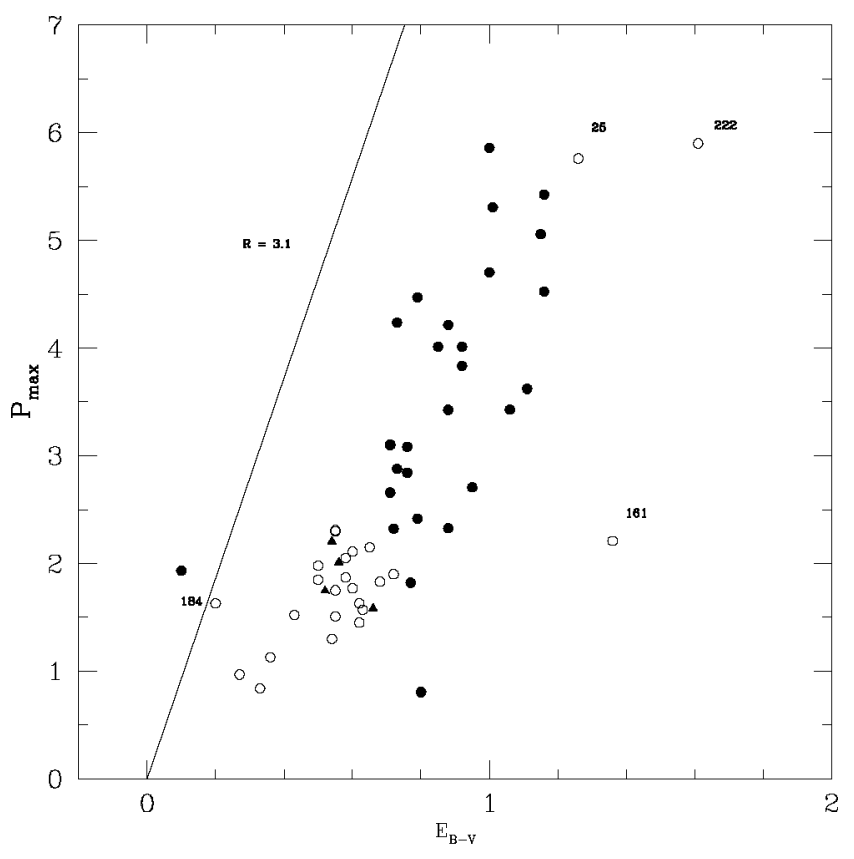

Fig. 3.- Polarization efficiency diagram. Filled symbols correspond to OVM00's observations, while open symbols are for stars observed in the direction of the halo. Triangles indicate the four stars adopted as front-side stars in the core investigation. The line of maximum efficiency is drawn adopting $R_{V}=3.1$.

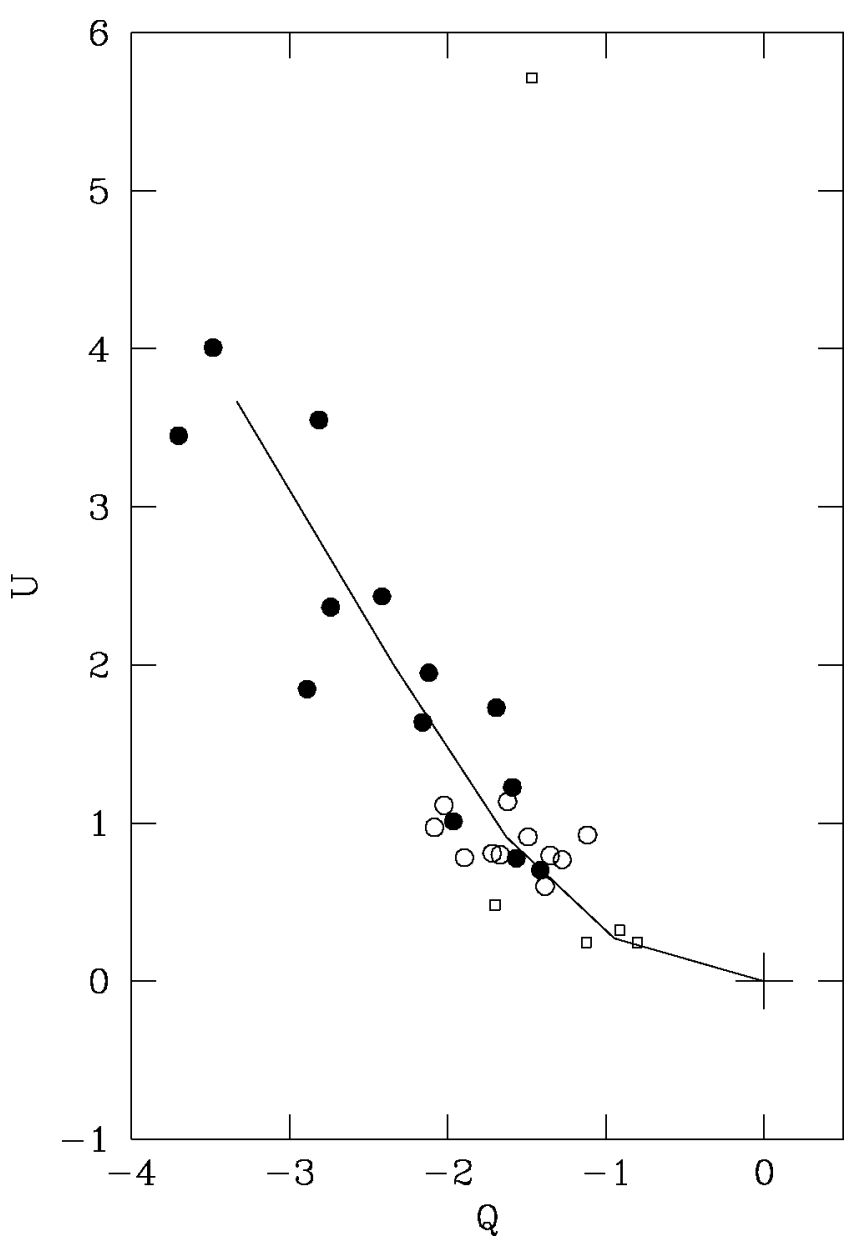

FIG. 4.- $Q$ - and $U$-parameters for the observed stars in NGC 6611. Filled circles are for observations in the direction of the core (OVM00), open circles are for halo stars (this investigation), and squares are for nonmembers from both works. Stars with intrinsic polarization are not included in this plot.

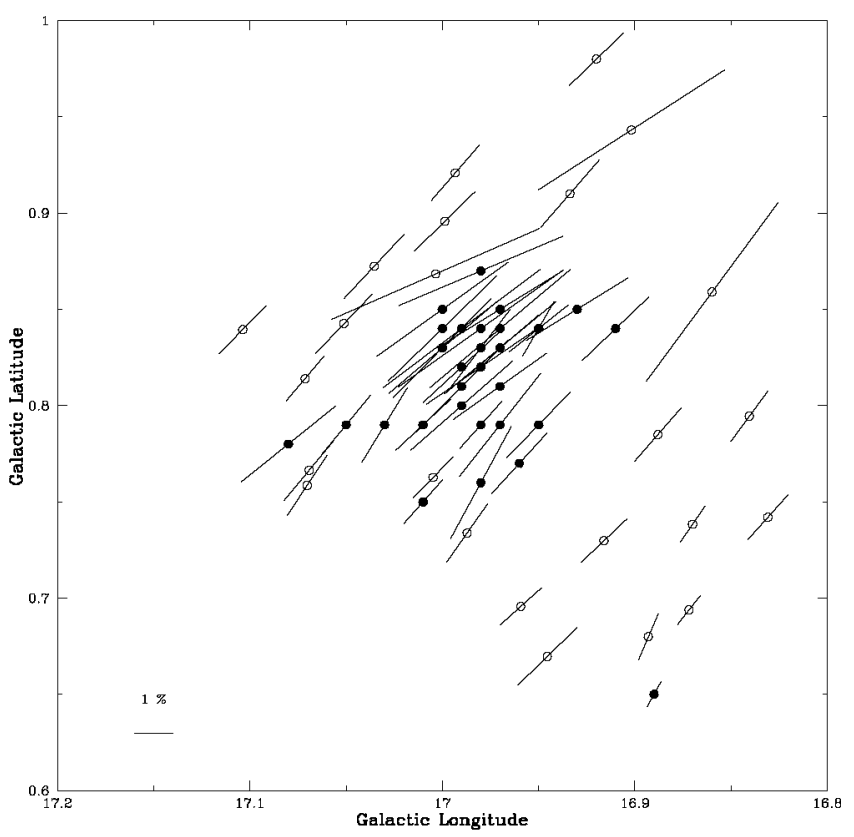

Fig. 5.-Polarization vectors and their orientations for the observed stars in the direction of NGC 6611. The symbols are the same as in previous figures. The length of each vector is proportional to the percentage polarization.

do not overlap each other in the plot; the only exceptions are stars $349,367,374$, and 455 , which were selected in the past investigation as front-side stars in order to subtract the dust in front of core stars. According to this plot, it seems that these four stars are halo members projected against the core. The light from the halo stars shows a mean vectorial polarization of $1.93 \% \pm 0.3 \%$. Their $\overline{\lambda_{\max }}$ has a value similar to the ISM $(0.55 \pm 0.07 \mu \mathrm{m})$, and the low dispersion indicates homogeneity in the distribution of the polarizing dust. The polarization vectors have a mean direction given by $\overline{\theta_{V}}=77^{\circ} .1 \pm 5^{\circ} .5$. Stars with intrinsic polarization have not been included in the calculations.

Star $103\left(E_{B-V}=0.44 \mathrm{mag}\right)$, plotted in Figure 4 as a square near the halo stars group, is considered a probable nonmember by de Winter et al. (1997), according to its excess. The star has a polarization of $1.78 \%$, and the orientation of its $e$-vector is $\theta_{V}=$ $82^{\circ} .1$. Both polarimetric magnitudes are compatible with halo membership, and because of this we propose that the star is a probable member of NGC 6611 but located near the cluster limits.

Finally, the filled symbols' distribution (Fig. 4) seems to reveal the presence of another group. We think they are only stars more deeply immersed in the dust core, or perhaps on the far side of it. The line in Figure 4 represents the changing direction of the projected $e$-vector, while the line of sight travels from the Sun to the cluster. As can be seen, there is practically no deviation in the line direction between halo and core stars.

Figure 5 plots the polarization vectors and their orientations for the observed stars in the direction of the cluster. It reproduces the upper plot of OVM00's Figure 6 for core stars, with the addition of halo stars ( filled and open circles, respectively). Position angles for both groups are generally similar, with a slight difference in mean direction of the $e$-vector between both groups, which is within the errors: $70^{\circ} .0 \pm 2.5$ (core) versus $73^{\circ} .7 \pm 2^{\circ} .5$ (halo).

Figure 6 shows the $E_{V-K} / E_{B-V}$ versus $\lambda_{\text {max }}$ plot for stars observed in the core of NGC 6611 (filled symbols) and in the halo (open symbols) following the procedure outlined in Hillenbrand et al. (1993) and Belikov et al. (1999). Here we find that the halo stars are possibly represented by the Whittet \& van Breda (1978; 


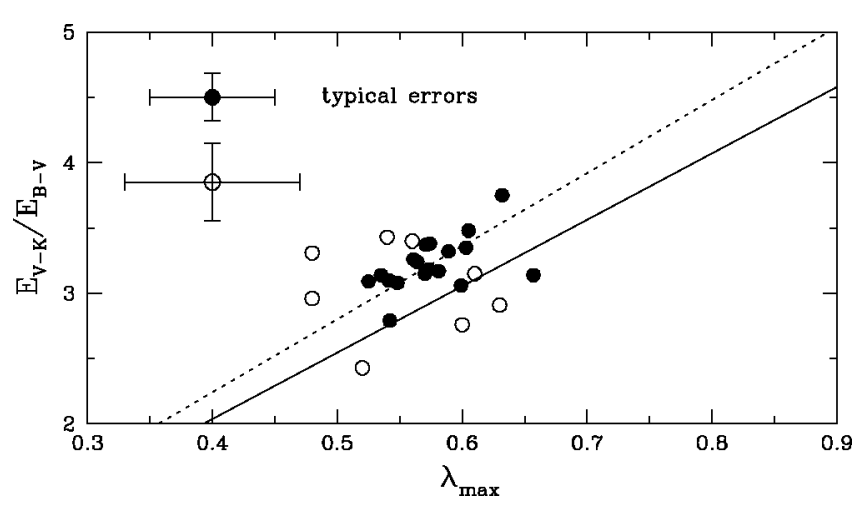

Fig. 6. $-E_{V-K} / E_{B-Y}$ vs. $\lambda_{\max }$ for stars observed in the core of NGC 6611 ( filled symbols) and its surroundings (open symbols). The lines show the Whittet \& van Breda (1978) relationship (solid line) and the relationship found in OVM00 for the core stars (dotted line).

$R_{V}=5.6 \lambda_{\max }$ ) relationship, but the somewhat larger errors involved prevent us from drawing definite conclusions.

\section{CONCLUSIONS}

We were not able to find any significant difference between the core and halo dust characteristics. Of the halo stars, $47 \%$ ( 8 out of 17) have indications of intrinsic polarization in their light, a percentage that is similar to what was found for core stars $(50 \%)$. A possible explanation for these high values comes from the work of Dûchene et al. (2001) on the total binary frequency of high-mass stars in NGC 6611. From observations of stars in the direction of the cluster (OVM00 and this paper) we have concluded that the light from member stars is affected by several dust clouds. Such dust clouds are located (1) in the Local arm, contributing to the final measured polarization by about $1.0 \%$; (2) in the Sagittarius arm, in front of the cluster outskirts (i.e., intra-arm dust, contributing another $0.8 \%$ ); and (3) variable intracluster dust contribution, as found in OVM00's core investigation and this paper.

We wish to acknowledge the technical support and hospitality at CASLEO during the observing runs. We also acknowledge the use of the Torino Photopolarimeter built at Osservatorio Astronomico di Torino (Italy) and operated under agreement between Complejo Astronómico El Leoncito and Osservatorio Astronomico di Torino. Special thanks go to Carlos Feinstein for valuable comments and to M. C. Fanjul de Correbo and Ruben Martínez for technical assistance.

Belikov, A. N., Kharchenko, N. V., Piskunov, A. E., \& Schilbach, E. 1999, A\&AS, 134,525

Chini, R., \& Wargau, W. F. 1990, A\&A, 227, 213

Clocchiati, A., \& Marraco, H. G. 1988, A\&A, 197, L1

de Winter, D., Koulis, C., Thé, P. S., van den Ancker, M. E., Perez, M. R., \& Bibo, E. A. 1997, A\&AS, 121, 223

Dûchene, G., Simon, T., Eisloffel, J., \& Bouvier, J. 2001, A\&A, 379, 147

Hillenbrand, L. A., Massey, P., Strom, S. E., \& Merrill, K. M. 1993, AJ, 106, 1906

Hiltner, W. A. 1956, ApJS, 2, 389

Hoag, A. A., Johnson, H. L., Iriarte, B., Mitchell, R. I., Hallam, K. L., \& Sharpless, S. 1961, Publ. USNO, 17, 345

Kumar, B., Sagar, R., Sanwall, B. B., \& Bessell, M. S. A. 2004, MNRAS, 353, 991

McMillan, R. S. 1978, ApJ, 225, 880

Orsatti, A. M., Vega, E. I., \& Marraco, H. G. 2000, A\&AS, 144, 195 (OVM00)

Scaltriti, F. 1994, The UBVRI Photopolarimeter of the Torino Astronomical Observatory (Tech. Publ. TP-001; Torino: Oss. Astron. Torino)

Schmidt-Kaler, Th. 1982, Landolt-Börnstein New Series, Group 6, Vol. 2b (Berlin: Springer)

Serkowski, K. 1973, in IAU Symp. 52, Interstellar Dust and Related Topics, ed. J. M. Greenberg \& H. C. van der Hulst (Dordrecht: Reidel), 145

Serkowski, K., Mathewson, D. L., \& Ford, V. L. 1975, ApJ, 196, 261

Tucholke, H. J., Geffert, M., \& Thé, P. S. 1986, A\&AS, 66, 311

Vega, E. I., Orsatti, A. M., \& Marraco, H. G. 1994, AJ, 108, 1834

Walker, M. F. 1961, ApJ, 133, 438

Whittet, D. C. B., Martin, P. G., Hough, J. H., Rouse, M. F., Bailey, J. A., \& Axon, D. J. 1992, ApJ, 386, 562

Whittet, D. C. B., \& van Breda, I. G. 1978, A\&A, 66, 57

Wilking, B. A., Lebofsky, M. J., Martin, P. G., Rieke, G. H., \& Kemp, J. C. 1980, ApJ, 235, 905 